\title{
Outpatient visit trends for internal medicine ambulatory care sensitive conditions after the COVID-19 pandemic: a time-series analysis
}

\author{
Ciara Pendrith ${ }^{1,2}$, Dhruv Nayyar ${ }^{1,3}$, Cherry Chu², Tara O'Brien ${ }^{1,4}$, Owen D. Lyons ${ }^{1,4}$, Payal Agarwal ${ }^{2,4,5}$,
}

Danielle Martin ${ }^{1,2,3,4,5}$, R. Sacha Bhatia ${ }^{1,2,3,4,6}$ and Geetha Mukerji, ${ }^{1,2,3,4^{*}}$ (B)

\begin{abstract}
Background: The COVID-19 pandemic led to a dramatic shift in the delivery of outpatient medicine with reduced in-person visits and a transition to predominantly virtual visits. We sought to understand trends in visit patterns for ambulatory care sensitive conditions (ACSCs) commonly seen in internal medicine clinics.

Methods: We included adult outpatients seen for an ACSC between March 15th, 2017 and March 14th, 2021 at a single-centre in Ontario, Canada. Monthly visits were assessed by visit type (new consultation, follow-up), diagnosis, and clinic. Time series analyses compared visit volumes pre- and post-pandemic. Proportion of virtual visits were compared before and during the pandemic. Patient and visit factors were compared between in-person and virtual visits.

Results: 8274 patients with 34,021 visits were included. Monthly visits increased by $15 \%$ during the pandemic $(p<0.0001)$. New consultations decreased by $10 \%(p=0.0053)$ but follow-up visits increased by $21 \%(p<0.0001)$. Monthly heart failure visits increased by $43 \%(p<0.0001)$ whereas atrial fibrillation visits decreased. Pre- pandemic, $<1 \%$ of visits were virtual compared to $82 \%$ during the pandemic $(p<0.0001)$. Less than half of heart failure visits were virtual whereas $>95 \%$ of diabetes visits were virtual.

Conclusions: We found a significant increase in overall visits to internal medicine clinics driven by increased volumes of follow-up visits, which more than offset decreased new consultations. There was variability in visit trends and uptake of virtual care by visit diagnosis, which may indicate challenges with delivery of virtual care for certain conditions.
\end{abstract}

Keywords: Virtual care, COVID-19 pandemic, Internal medicine, Ambulatory care sensitive conditions, Health services

\section{Background}

The COVID-19 pandemic led to rapid changes in the delivery of healthcare services to meet patient needs throughout the pandemic while reducing the risk of

\footnotetext{
*Correspondence: Geetha.Mukerij@wchospital.ca
}

${ }^{2}$ Women's College Hospital Institute for Health System Solutions \& Virtual

Care, 76 Grenville Street, Toronto, Ontario M5S 1B3, Canada

Full list of author information is available at the end of the article exposure to SARS-CoV-2. Among other adaptations, healthcare systems rapidly transitioned from traditional in-person office-based visits to predominantly virtual care [1-4].

Within Ontario, a Canadian province with a population of 14,500,000 and universal health insurance, adoption of virtual care was low before the pandemic $[5,6]$. Barriers included challenges with reimbursement, policy issues, and limited access to technology [1, 7-9]. Within Ontario 
specifically, virtual delivery of physician services were previously only covered by the Ontario Health Insurance Plan if delivered from a government-approved facility using a government-approved telemedicine platform $[3,5]{ }^{3,5}$ After the declaration of COVID-19 as a global pandemic, new temporary billing codes were issued on March 14th, 2020, which allowed physicians to be renumerated for outpatient visits conducted via telephone or videoconference at the same rate as an in-person visit which facilitated the widespread adoption of virtual care $[3,10]$. While previous studies have examined changes in visit volumes and uptake of virtual care during the pandemic, these have generally been limited to primary care $[4,5,10]$ or narrow scopes of care $[11,12]$.

To our knowledge, no study to date has assessed the COVID-19 pandemic's impact on visit trends and utilization of virtual care across multiple internal medicine ambulatory care sensitive conditions (ACSCs). ACSCs are health conditions where high quality and timely care may prevent acute exacerbation, sequelae of disease, and emergency department visits or hospitalizations [13]. By assessing changes in outpatient visits for several ACSCs across a single institution, we can understand the relative impact of the pandemic and virtual care on different conditions. These findings may highlight areas where virtual care is better suited than others. Additionally, understanding care patterns for multiple ACSCs may help organizations plan future allocation of resources within an evolving healthcare environment in the ongoing pandemic with the goals of preventing clinical deterioration and avoidable inpatient visits.

Our objectives were to understand how the onset of the COVID-19 pandemic and introduction of virtual care billing codes impacted patterns of care and uptake of virtual care for outpatients with common internal medicine ACSCs at a large academic ambulatory hospital in Ontario, Canada. Furthermore, we sought to understand patient and visit factors associated with utilization of virtual care.

\section{Methods}

\section{Design, setting and participants}

We conducted a repeated cross-sectional study of all patients seen in internal medicine clinics for an ACSC at Women's College Hospital (WCH) in Toronto, Ontario between September 15th, 2017 and March 14th, 2021. $\mathrm{WCH}$ is an ambulatory care hospital affiliated with the University of Toronto.

Internal medicine clinics included were the following: General Internal Medicine (GIM) clinic; Acute Ambulatory Care Unit, which is a short stay $(<24 \mathrm{~h})$ unit providing urgent assessment, and treatment of internal medicine conditions; Cardiology clinic; Atrial Fibrillation clinic; General Endocrinology clinic; Diabetes clinic; and Respirology clinic. ACSCs included in this study were hypertension, congestive heart failure, coronary artery disease, atrial fibrillation, diabetes mellitus, asthma, and chronic obstructive pulmonary disease [13].

Patient visits meeting the following criteria were included: 1) main visit diagnosis was an ACSC to an internal medicine clinic; and 2) visit occurred during the study time period. Visits were included if the healthcare provider was a physician, nurse practitioner, or nurse. Both new consultations and follow-up visits were included. Patient encounters were excluded if the encounter was for a procedure with no corresponding office or virtual care visit. Multiple same day visits to different providers were excluded if the encounter ID was the same (e.g. seen by physician and nurse for the same encounter).

The study period is divided into pre-pandemic and pandemic periods which are defined as September 15th, 2017-March 14th, 2020 and March 15th, 2020-March 14th, 2021, respectively. The study period was selected to include two and a half years of data before and one year after the introduction of temporary virtual care billing codes on March 14th, 2020 to allow sufficient data points to adjust for pre-pandemic trends in the time series analysis.

\section{Data sources}

Data for this study were collected from the hospital's electronic health record EPIC. Data on visit information and patient characteristics were extracted electronically using a data query, and a manual chart review was performed for any missing or unknown variables. Video visits were delivered through Zoom videoconferencing technology [14] integrated through the electronic medical record patient portal myHealthRecord [15].

\section{Baseline characteristics}

For each included visit we extracted the visit date, main diagnosis using the diagnostic code, clinic, visit type (new consultation, follow-up visit), and visit modality (in-person, or virtual including telephone and videoconference visits). The following baseline patient characteristics were captured at the time of each visit: age, sex, and whether the patient had an email registered.

\section{Outcomes}

Our primary outcome was change in average monthly visits after the onset of the pandemic and introduction of virtual care billing codes. We assessed changes in visit volumes overall, as well as by visit type to understand changes in number of new consultations and follow-up visits. Visit volumes by visit diagnoses were compared 
to understand differences in the types of patient conditions being seen. Additionally, we assessed the proportion of virtual visits in the pre-pandemic and pandemic periods. Secondary outcomes included patient and visit factors associated with virtual care vs. in-person visits in the pandemic period.

\section{Statistical analyses}

Baseline characteristics of patients and visits were compared before and during the pandemic using Chi-square tests and t-tests. Average monthly visit numbers were compared during the pre-pandemic and pandemic periods using two-sample t-tests and the change in monthly visits was calculated as both the mean and relative difference. To understand differences in pandemic visit volumes compared with historical data, time series analyses were performed using interventional Autoregressive Integrated Moving Average (ARIMA) models to examine the statistical significance of the change in visit volumes from pre-pandemic to during. Models were run for total visits, new consultations, follow-ups, and each visit diagnosis. Interventional ARIMA modelling is commonly used to analyze the effect of change (e.g. major event or intervention) in a time-series analysis [16]. A step function was applied to the model to characterize the start of the pandemic. Secondary outcomes of factors associated with virtual visits in the pandemic period were assessed using Chi-square tests and two-sample t-tests. $P$-values $<0.05$ were considered statistically significant. All statistical analyses were completed using Microsoft Excel and SAS version 9.4.

\section{Ethics}

Ethics approval was received from the Research Ethics Board at Women's College Hospital (REB approval \#: 2019-0191-E).

\section{Results}

\section{Study participants and visits}

A total of 8274 unique patients with 34,021 outpatient visits were included in the study. Most patients had multiple visits during the study time period, with $28.2 \%$ having one visit, $16.9 \%$ having two visits, and $54.9 \%$ having three or more visits. Characteristics of patients and their visits are included in Table 1 . The mean age was 58.1 years and $61.4 \%$ of visits were delivered to female patients; $52.3 \%$ of patients had an email registered. There were no differences in patient demographics in the prepandemic and pandemic periods.

There were 23,279 and 10,742 visits in the pre-pandemic and pandemic periods, respectively. The proportion of new consultation visits decreased from 17.2\% pre-pandemic to $13.4 \%$ during the pandemic $(p<0.0001)$.
Pre-pandemic, $99.9 \%$ of visits were conducted in-person with only 18 virtual visits, all of which were telephone visits for diabetes patients seen in the General Endocrinology and Diabetes clinics. In the pandemic period, the proportion of in-person visits decreased to $18.1 \%$ $(p<0.0001)$ with the majority of virtual visits being conducted over telephone $(95.3 \%)$ and a minority by videoconference (4.7\%).

\section{Visit trends}

Figure 1 displays monthly overall visits, new consultations, and follow-up visits across the study period. Results from the time series analysis are shown in Table 2. Average monthly overall visits decreased by $15 \%$ between the pre-pandemic and pandemic periods (776 vs. 895), new consultations decreased by $10 \%$ (134 vs. 120 ), and followups increased by $21 \%$ (642 vs. 776 ). The changes in visit volumes were significant for all types of visits: overall visits $(p<0.0001)$, new consultations (0.0053), and followups $(\mathrm{p}<0.0001)$.

Average monthly visits by visit diagnosis are displayed in Fig. 2. Monthly visits for atrial fibrillation decreased by $18 \%$ ( 60 vs. 49$)$ while congestive heart failure visits increased by $43 \%$ (44 vs. 63 ). The change in visit volumes was significant for both atrial fibrillation $(p=0.013)$ and congestive heart failure $(p<0.0001)$. There were increases in monthly visits for hypertension (20\%), diabetes (16\%), and asthma (23\%). The increases for these conditions were significant in the ARIMA model. There was no significant difference in monthly visits for coronary artery disease or chronic obstructive pulmonary disorder.

Figure 3 shows the proportion of visits by visit modality in the pandemic period. The second month of the pandemic (April 2020) saw the lowest monthly proportion of in-person visits at $7 \%$ after which in-person visits gradually increased until September 2020, when $28 \%$ of monthly visits were in-person. After September 2020, the proportion of in-person visits decreased to $18-23 \%$ per month.

\section{Factors associated with virtual visits in the pandemic period}

Table 3 shows patient and visit factors by visit modality after the onset of the pandemic. Patients seen virtually during the pandemic were significantly younger than those seen in-person (mean age 64.9 seen in-person vs. 56.6 seen virtually, $p<0.0001$ ). Male patients were more likely to be seen in-person with $20.2 \%$ of visits by male patients being in-person compared to $16.7 \%$ of visits by female patients $(p<0.0001)$. Patients with an email registered with the hospital were more likely to be seen virtually than those without an email on file $(85.7 \%$ vs. $76.7 \%$, $\mathrm{p}<0.0001$ ). 
Table 1 Baseline characteristics of patients and visits in the pre-pandemic and pandemic periods

\begin{tabular}{|c|c|c|c|c|}
\hline & $\begin{array}{l}\text { Pre-pandemic } \\
n=23,279\end{array}$ & $\begin{array}{l}\text { Pandemic } \\
n=10,742\end{array}$ & $\begin{array}{l}\text { Overall } \\
n=34,021\end{array}$ & $p$-value \\
\hline \multicolumn{5}{|l|}{ Patient characteristics } \\
\hline Mean age (SD) & $58.1(18.9)$ & $58.1(19.0)$ & $58.1(18.9)$ & 0.99 \\
\hline Sex & & & & 0.35 \\
\hline Female & $14,331(61.6 \%)$ & $6556(61.0 \%)$ & $20,887(61.4 \%)$ & \\
\hline Male & $8948(38.4 \%)$ & $4186(39.0 \%)$ & $13,134(38.6 \%)$ & \\
\hline Email registered & $11,650(50.0 \%)$ & $6151(57.3 \%)$ & $17,801(52.3 \%)$ & $<0.0001$ \\
\hline \multicolumn{5}{|l|}{ Visit characteristics } \\
\hline Visit type & & & & $<0.0001$ \\
\hline New consultation & $4007(17.2 \%)$ & $1436(13.4 \%)$ & $5443(16.0 \%)$ & \\
\hline Follow-up & $19,272(82.8 \%)$ & $9306(86.6 \%)$ & $28,578(84.0 \%)$ & \\
\hline Visit modality (grouped) & & & & $<0.0001$ \\
\hline In-person & 23,261 (99.9\%) & 1943 (18.1\%) & $25,204(74.1 \%)$ & \\
\hline Virtual care & $18(0.1 \%)$ & $8799(81.9 \%)$ & $8817(25.9 \%)$ & \\
\hline Visit modality (specified) & & & & $<0.0001$ \\
\hline In-person & 23,261 (99.9\%) & 1943 (18.1\%) & $25,204(74.1 \%)$ & \\
\hline Telephone & $18(0.1 \%)$ & $8388(78.1 \%)$ & $8406(24.7 \%)$ & \\
\hline Videoconference & $0(0.0 \%)$ & $411(3.8 \%)$ & $411(1.2 \%)$ & \\
\hline Visit diagnosis & & & & $<0.0001$ \\
\hline AFib & $1797(7.7 \%)$ & $590(5.5 \%)$ & $2387(7.0 \%)$ & \\
\hline$C A D$ & 2097 (9.0\%) & $898(8.4 \%)$ & 2995 (8.8\%) & \\
\hline $\mathrm{CHF}$ & $1319(5.7 \%)$ & $752(7.0 \%)$ & $2071(6.1 \%)$ & \\
\hline HTN & $3285(14.1 \%)$ & $1571(14.6 \%)$ & $4856(14.3 \%)$ & \\
\hline DM & $10,953(47.1 \%)$ & $5088(47.4 \%)$ & $16,041(47.2 \%)$ & \\
\hline Asthma & $2732(11.7 \%)$ & 1340 (12.5\%) & 4072 (12.0\%) & \\
\hline COPD & 1096 (4.7\%) & $503(4.7 \%)$ & 1599 (4.7\%) & \\
\hline Clinic & & & & $<0.0001$ \\
\hline AACU & 1758 (7.6\%) & 905 (8.4\%) & $2663(7.8 \%)$ & \\
\hline AFib clinic & $663(2.8 \%)$ & 115 (1.1\%) & 778 (2.3\%) & \\
\hline Cardiology clinic & $6012(25.8 \%)$ & 2768 (25.8\%) & $8780(25.8 \%)$ & \\
\hline GIM clinic & $655(2.8 \%)$ & $248(2.3 \%)$ & 903 (2.7\%) & \\
\hline Endocrine clinic & 8070 (34.7\%) & 3836 (35.7\%) & 11,906 (35.0\%) & \\
\hline DM clinic & $2428(10.4 \%)$ & 1066 (9.9\%) & 3494 (10.3\%) & \\
\hline Respirology clinic & 3693 (15.9\%) & 1804 (16.8\%) & 5497 (16.2\%) & \\
\hline
\end{tabular}

AFib atrial fibrillation, CAD coronary artery disease, CHF congestive heart failure, HTN hypertension, DM diabetes mellitus, COPD chronic obstructive pulmonary disorder, AACU Acute Ambulatory Care Unit, GIM general internal medicine

New consultations were more likely to be seen inperson (33.5\%) than follow-up visits $(15.7 \%, \mathrm{p}<0.0001)$. There were large differences in the proportion of virtual visits by clinic location in the pandemic period. Over $85 \%$ of visits to the Diabetes, General Endocrinology, General Internal Medicine, and Cardiology clinics were conducted virtually after the pandemic onset. In contrast, visits to the Acute Ambulatory Care Unit and Atrial Fibrillation clinics were mostly in-person (10.1 and 27.0\% virtual visits, respectively). Amongst congestive heart failure visits, $48.1 \%$ were seen virtually. Greater than $65 \%$ of visits for all other diagnoses were seen virtually. The most common conditions seen virtually were coronary artery disease (86.6\%) and diabetes (95.5\%).

\section{Discussion}

In this analysis of outpatient visits to internal medicine clinics for ACSCs at a large ambulatory hospital in Ontario, Canada, we found a dramatic shift towards virtual care after the onset of the COVID-19 pandemic, as well as several changes in visit trends for overall visits and by specific visit diagnoses. While new consultations decreased by $10 \%$ during the pandemic, this was offset by an increase in follow-up visits leading to an overall $15 \%$ 


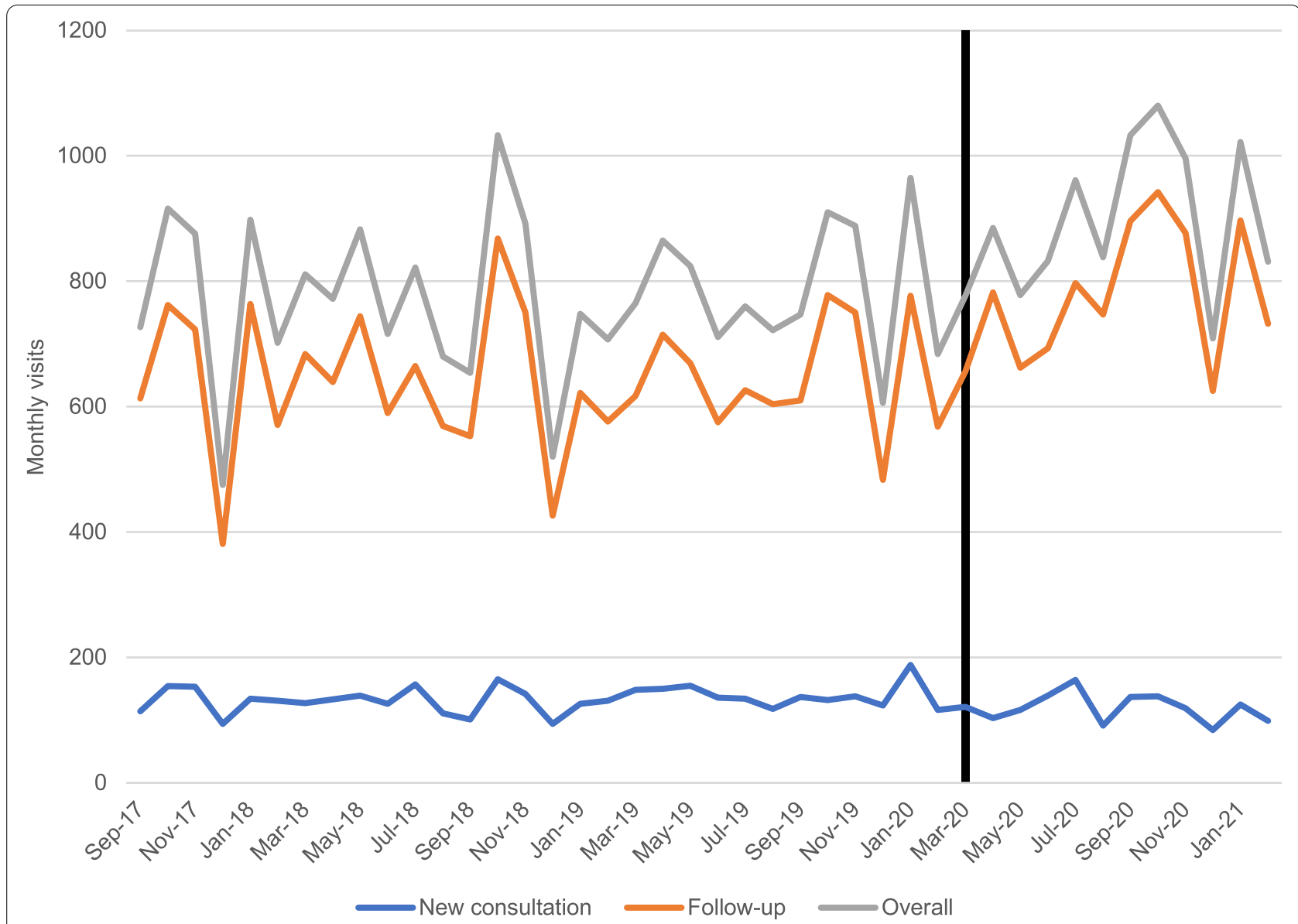

Fig. 1 Average monthly overall, new consultation, and follow-up visits. The black vertical line represents the start of the COVID-19 pandemic

Table 2 Average monthly visits in the pre-pandemic and pandemic periods

\begin{tabular}{|c|c|c|c|c|c|}
\hline & $\begin{array}{l}\text { Pre-pandemic mean visits } \\
\text { ( } \pm \text { SD) }\end{array}$ & $\begin{array}{l}\text { Pandemic mean visits } \\
( \pm \text { SD) }\end{array}$ & Mean difference & Relative difference & $p$-value* \\
\hline Overall visits & $776( \pm 126)$ & $895( \pm 120)$ & +119 & $+15 \%$ & $<0.0001$ \\
\hline New consults & $134( \pm 21)$ & $120( \pm 23)$ & -14 & $-10 \%$ & 0.0053 \\
\hline Follow-up visits & $642( \pm 109)$ & $776( \pm 108)$ & +133 & $+21 \%$ & $<0.0001$ \\
\hline AFib visits & $60( \pm 14)$ & $49( \pm 9)$ & -11 & $-18 \%$ & 0.013 \\
\hline CAD visits & $70( \pm 19)$ & $75( \pm 14)$ & +5 & $+7 \%$ & 0.42 \\
\hline CHF visits & $44( \pm 8)$ & $63( \pm 17)$ & +19 & $+43 \%$ & $<0.0001$ \\
\hline HTN visits & $110( \pm 20)$ & $131( \pm 27)$ & +21 & $+20 \%$ & 0.0049 \\
\hline DM visits & $365( \pm 57)$ & $424( \pm 58)$ & +59 & $+16 \%$ & $<0.0001$ \\
\hline Asthma visits & $91( \pm 23)$ & $112( \pm 23)$ & +21 & $+23 \%$ & $<0.0001$ \\
\hline COPD visits & $37( \pm 10)$ & $42( \pm 8)$ & +5 & $+15 \%$ & 0.10 \\
\hline
\end{tabular}

AFib atrial fibrillation, CAD coronary artery disease, CHF congestive heart failure, $H T N$ hypertension, $D M$ diabetes mellitus, COPD chronic obstructive pulmonary disorder 


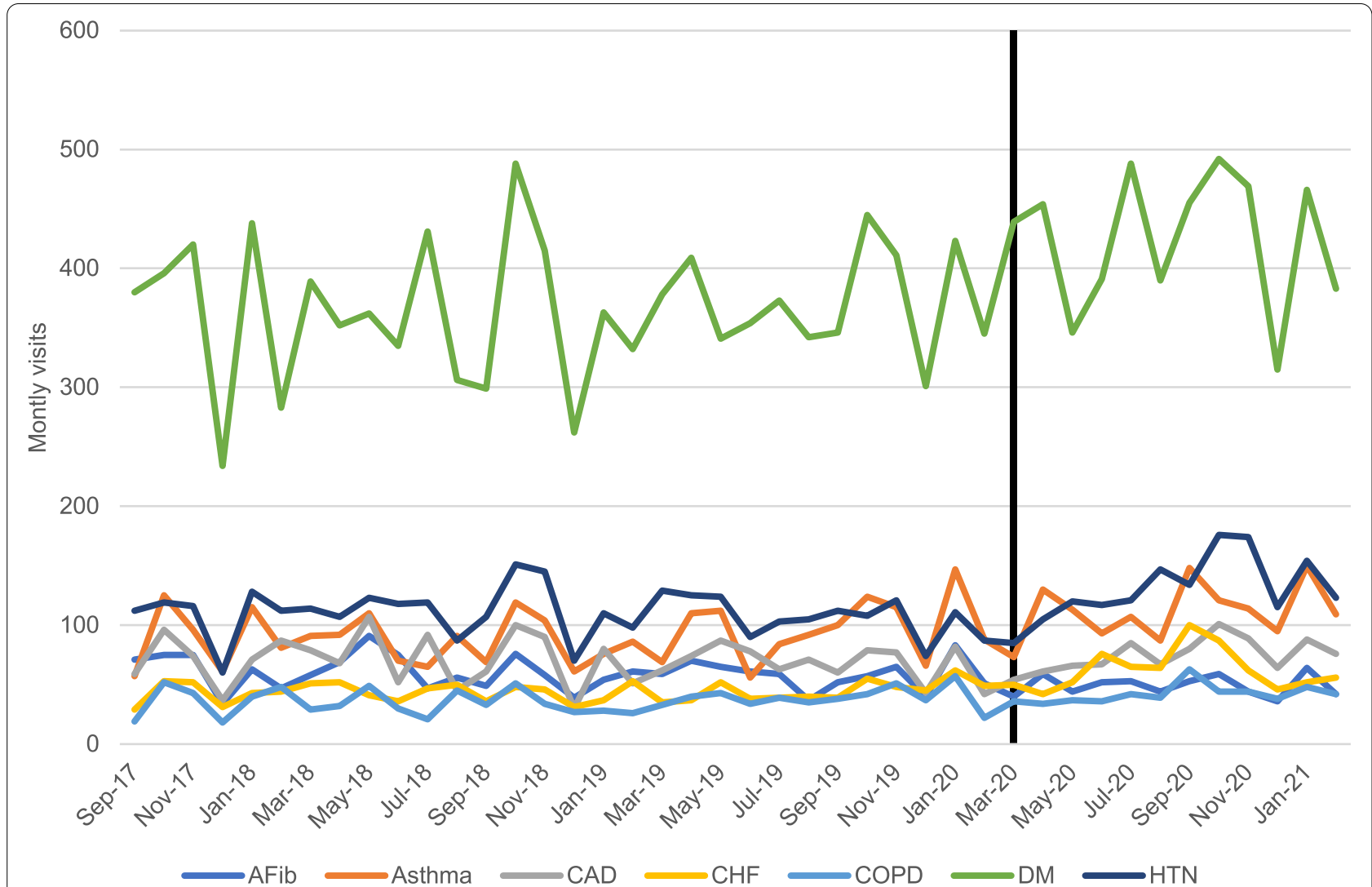

Fig. 2 Average monthly visits by visit diagnosis. The black vertical line represents the onset of the COVID-19 pandemic. AFib=atrial fibrillation, $\mathrm{CAD}=$ coronary artery disease, $\mathrm{COPD}=$ chronic obstructive pulmonary disorder, $\mathrm{DM}=$ diabetes mellitus, $\mathrm{HTN}=$ hypertension

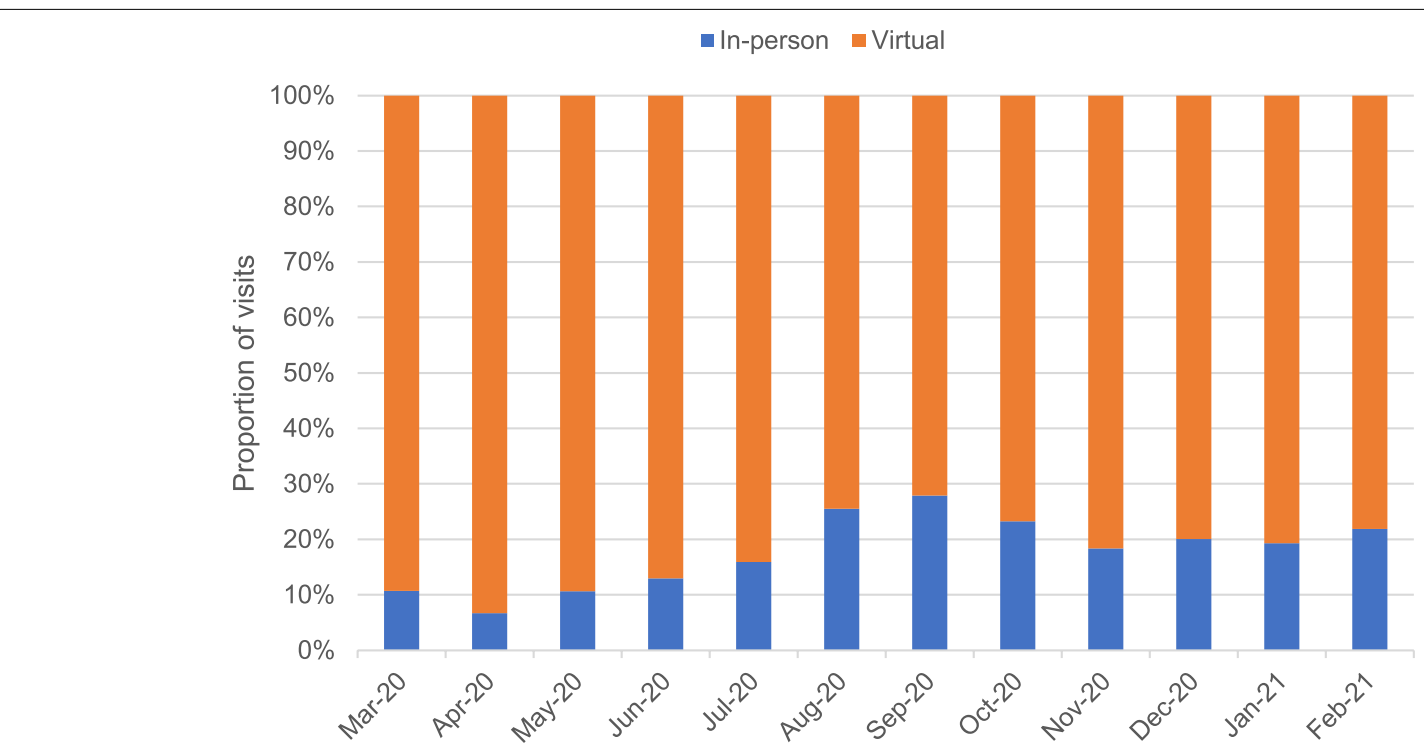

Fig. 3 Visit modality proportions by study month in the pandemic period 
Table 3 Patient and visit characteristics by visit modality in the pandemic period

\begin{tabular}{|c|c|c|c|c|}
\hline & $\begin{array}{l}\text { In-person } \\
\text { visits }\end{array}$ & Virtual visits & Overall & $p$-value \\
\hline $\mathrm{N}$ & 1943 & 8799 & 10,742 & \\
\hline Mean age (SD) & $64.9(18.0)$ & $56.6(18.9)$ & $58.1(19.0)$ & $<0.0001$ \\
\hline Sex & & & & $<0.0001$ \\
\hline Female & $1096(16.7 \%)$ & $5460(83.3 \%)$ & $6556(100 \%)$ & \\
\hline Male & 847 (20.3\%) & 3339 (79.8\%) & 4186 (100\%) & \\
\hline $\begin{array}{l}\text { Email regis- } \\
\text { tered }\end{array}$ & & & & $<0.0001$ \\
\hline Yes & 875 (14.2\%) & $5276(85.8 \%)$ & 6151 (100\%) & \\
\hline No & $1068(23.3 \%)$ & 3523 (76.7\%) & 4591 (100\%) & \\
\hline Visit type & & & & $<0.0001$ \\
\hline $\begin{array}{l}\text { New consulta- } \\
\text { tion }\end{array}$ & 481 (33.\%) & 955 (66.5\%) & $1436(100 \%)$ & \\
\hline Follow-up & $1462(15.7 \%)$ & 7844 (84.3\%) & 9306 (100\%) & \\
\hline Visit diagnosis & & & & $<0.0001$ \\
\hline AFib & 200 (33.9\%) & 390 (66.1\%) & 590 (100\%) & \\
\hline$C A D$ & 120 (13.4\%) & 778 (86.6\%) & 898 (100\%) & \\
\hline $\mathrm{CHF}$ & 390 (51.9\%) & 362 (48.1\%) & 752 (100\%) & \\
\hline HTN & 377 (24.0\%) & 1194 (76.0\%) & 1571 (100\%) & \\
\hline $\mathrm{DM}$ & $231(4.5 \%)$ & 4857 (95.5\%) & $5088(100 \%)$ & \\
\hline Asthma & 469 (35.0\%) & 871 (65.0\%) & 1340 (100\%) & \\
\hline COPD & $156(31.0 \%)$ & 347 (69.0\%) & $503(100 \%)$ & \\
\hline Clinic & & & & $<0.0001$ \\
\hline AACU & 814 (89.9\%) & 91 (10.1\%) & 905 (100\%) & \\
\hline AFib clinic & $84(73.0 \%)$ & $31(27.0 \%)$ & 115 (100\%) & \\
\hline $\begin{array}{l}\text { Cardiology } \\
\text { clinic }\end{array}$ & 311 (11.2\%) & 2457 (88.8\%) & 2768 (100\%) & \\
\hline GIM clinic & 19 (7.7\%) & 229 (92.3\%) & 248 (100\%) & \\
\hline $\begin{array}{l}\text { Endocrine } \\
\text { clinic }\end{array}$ & 68 (1.8\%) & 3768 (98.2\%) & $3836(100 \%)$ & \\
\hline DM clinic & $50(4.7 \%)$ & 1106 (95.3\%) & 1066 (100\%) & \\
\hline $\begin{array}{l}\text { Respirology } \\
\text { clinic }\end{array}$ & 597 (33.1\%) & 1207 (66.9\%) & 1804 (100\%) & \\
\hline
\end{tabular}

AFib atrial fibrillation, $C A D$ coronary artery disease, $C H F$ congestive heart failure, HTN hypertension, DM diabetes mellitus, COPD chronic obstructive pulmonary disorder, AACU Acute Ambulatory Care Unit, GIM general internal medicine

increase in visits. Our results showed a transition from primarily in-person visits pre-pandemic to predominantly virtual care during the pandemic. Importantly, there were several factors associated with visit modality in the pandemic period, with patients who were older, men, and without a registered email account more likely to be seen in-person than virtually. There were also significant differences in uptake of virtual care by clinic and visit diagnosis, with less than half of heart failure visits being seen virtually, in contrast to diabetes care which was nearly entirely virtual.

The dramatic shift towards virtual care during the COVID-19 pandemic has been consistently shown in other studies across a number of jurisdictions, which is consistent with our findings [1-5, 10-12, 17-19]. A study of visit trends to Veterans Affairs clinics in the United States during the first 10 weeks of the pandemic showed a decrease in in-person visits by $56 \%$ which was partially offset by an increase in telephone and video visits, but overall visits still decreased by $30 \%$ [1]. Similarly, other studies from the US and Canada have shown an overall decline in visit numbers after the pandemic $[10,20,21]$. These findings are in contrast to our findings of a significant increase in patient volumes during the pandemic after accounting for prior trends, which was driven by more frequent follow-up visits. The reasons for these differences in findings are unclear and may be due to our study having a longer follow-up period allowing for a correction in visits after an initial drop with the start of the pandemic or due to differences in populations and conditions studied.

A prior study demonstrated that new consultations were postponed while follow-up visits shifted to primarily telemedicine during the pandemic [22], which is consistent with our findings, but the literature on patterns in internal medicine is lacking. We do not have data on the reasons leading to decreased new consultations and increased follow-up visits; however, there are several potential factors. Decreased visits in primary care during the pandemic may have led to decreased referrals as demonstrated in Ontario [10] and other jurisdictions during the first wave of the pandemic [2325]. Other reported barriers, such as patient concerns about acquiring COVID-19 leading to delay seeking care [26] and decreased access to care [27] may have also impacted new visits. Regarding increased followup visits, telephone visits may have facilitated easier access to follow-up visits with a prior study showing that virtual follow-up visits were more efficient $[28,29]$. Similarly, both patients and providers have reported feeling telemedicine visits worked best for follow-up visits [30]. Furthermore, with the introduction of billing codes for telephone visits, physicians may be booking more follow-up telephone visits for brief calls that would have previously occurred but not been remunerated. Increased follow-up visits may be due to physicians wanting closer follow-up because they are unable to examine their patients, patients seeking specialist care rather than going to emergency departments for exacerbations of their chronic illness, decreased patient access to primary care [10] leading to more frequent telephone visits with specialists, or physicians feeling more comfortable asking patients for frequent followups because of the convenience of telemedicine [29]. Finally, delays in access to tests and procedures during the pandemic [27] may have led to patients needing 
to rebook earlier follow-up after completing delayed investigations.

To our knowledge, our study is the first to examine how visits for a range of common internal medicine clinic conditions changed during the pandemic. We found variable uptake of virtual care across different clinics and conditions. Patient visits for CHF, hypertension, and asthma each increased by $20 \%$, whereas atrial fibrillation visits decreased by nearly $20 \%$. We cannot discern the reasons for the variability by condition but there are several potential patient, provider, and system factors that may contribute. Our finding that patients who were older were less likely to use virtual care is consistent with past studies, $[12,21,31,32]$ as well as those showing that older patients are less likely to have internet and are slower to adopt technology [33-35]. Also, sicker or older patients may require more frequent follow-up. For example, CHF patients who are at risk of exacerbation may need closer follow-up to remotely monitor for signs of exacerbation with reduced in-person visits for volume status exams. Similarly, hypertension may lend itself easily to virtual follow-up if patients monitor their blood pressure at home allowing for quick telephone visits to titrate medications [29]. Female patients were more likely to be seen virtually in our study, although findings from other studies on sex differences in virtual care uptake have been mixed [10, 21, 31]. In terms of provider factors, a prior study showed that physicians viewed virtual care as optimally suited for managing conditions that primarily involved counselling and were less reliant on physical exams, and in particular, that hypertension and diabetes were easily managed virtually [29]. Other provider factors that may contribute to variability include familiarity or lack thereof with virtual mediums or supplier-induced demand. Finally, system factors likely contributed to decreased atrial fibrillation visits because of decreased clinic hours and fewer presentations to the emergency department, which is the primary referral mechanism to this clinic.

There are several limitations of our study. First, this was a single centre study of select internal medicine clinics and conditions at an academic hospital under a universal healthcare system in Canada. Our findings may not be generalizable to other conditions or health care systems. Second, visit diagnoses were based on the most responsible diagnoses coded by the visit physician, and for some patients, multiple conditions could have been addressed in a single visit. It is possible that some patients followed for multiple conditions by the same provider (e.g. atrial fibrillation and heart failure) had varying visit diagnoses across different visits despite the same conditions repeatedly being addressed in the same visit. This may contribute to the trends in conditions seen in the pre-pandemic and pandemic periods. However, we expect this to affect a minority of patients and not substantially alter our findings. Third, we were unable to extract data on comorbidities and sociodemographic factors that may be associated with uptake of virtual care such as high disease burden, ethnicity, language, socioeconomic status, and underhousing. Other studies from the US have shown these factors to be associated with telemedicine use [21, 31, 36, 37]; however we were limited to data available within the EMR. Finally, while this study examined trends in outpatient internal medicine visits and uptake of virtual care during the pandemic, we did not examine quality of care, outcomes, or patient satisfaction.

\section{Conclusions}

The COVID-19 pandemic led to a transition in the delivery of healthcare in Ontario from almost entirely in-person visits to over $80 \%$ virtual care in a short time period. Virtual care remained the predominant model of care delivery a year after the onset of the pandemic, and there were changes in the types of office visits and visit diagnoses seen during the pandemic. Several patient and visit factors were associated with greater uptake of virtual care. This study contributes to the growing literature on the use of virtual care and the effect of the pandemic on health services delivery. Future research is needed to understand drivers at the patient, provider, and system level led to decreased new consultations and increased follow-up visits. Data from other centres is needed to understand if our observation that patients with certain conditions were more likely to be seen virtually is replicated and understand the drivers behind these differences. Similarly, future research is needed on the equity of virtual care and how certain groups may be disadvantaged by the rapid shift towards virtual care. Finally, given the persistence of virtual care one year from pandemic onset as well as the multiple extensions of the temporary billing codes by the Ontario government, we suspect that virtual care will continue to be utilized in the future as part of the physician's toolbox to provide quality care. However, this speaks to the importance of future work to understand the impact of virtual care on quality of care, patient outcomes, patient satisfaction, and cost-effectiveness of outpatient care.

\section{Abbreviations}

COVID-19: Coronavirus disease 2019; SARS-CoV-2: Severe acute respiratory syndrome coronavirus 2; ASCS: Ambulatory care sensitive condition; WCH: Women's College Hospital; ARIMA: Autoregressive Integrated Moving Average.

\section{Acknowledgements}

The authors would like to thank Hayley Baranek, Patricia Rios, Xiao Zhou, Hye Rin Kim, Women's Virtual Operations team, Department of Medicine at Women's College Hospital for their support of this work. Dr. Payal Agarwal 
is supported by a New Investigator Award from the Department of Family Medicine, University of Toronto.

\section{Authors' contributions}

$\mathrm{CP}, \mathrm{DN}, \mathrm{CC}, \mathrm{TO}, \mathrm{ODL}, \mathrm{RSB}, \mathrm{GM}$ contributed to the design of the study. CP and CC contributed to the analysis of data. CP, DN, CC, TO, ODL, PA, DM, RSB, GM contributed to the interpretation of the data, drafting and critical revision of the manuscript. All authors read and approved the final version of the manuscript.

\section{Funding}

This research received no specific grant from any funding agency in the public, commercial or not-for-profit sectors. We would like to acknowledge Women's College Hospital Institute for Health System Solutions \& Virtual Care (WIHV) for in-kind resources provided.

\section{Availability of data and materials}

Available on request from Dr. Geetha Mukerji. There were no public or administrative databases used for this project. The sole data source for this project was the Electronic Medical Record at Women's College Hospital, Epic. The Women's College Hospital Research Ethics Board approved access.

\section{Declarations}

\section{Ethics approval and consent to participate}

Ethics approval was received from the Research Ethics Board at Women's College Hospital (REB approval \#: 2019-0191-E).

\section{Consent for publication}

Not applicable.

\section{Competing interests}

None to declare.

\section{Author details}

${ }^{1}$ Temerty Faculty of Medicine, University of Toronto, Toronto, ON, Canada. ${ }^{2}$ Women's College Hospital Institute for Health System Solutions \& Virtual Care, 76 Grenville Street, Toronto, Ontario M5S 1B3, Canada. ${ }^{3}$ Institute of Health Policy, Management and Evaluation, Dalla Lana School of Public Health, University of Toronto, Toronto, ON, Canada. ${ }^{4}$ Women's College Hospital, Toronto, ON, Canada. ${ }^{5}$ Department of Family and Community Medicine, University of Toronto, Toronto, ON, Canada. ${ }^{6}$ Peter Munk Cardiac Centre, University Health Network, Toronto, ON, Canada.

Received: 27 September 2021 Accepted: 31 January 2022

Published online: 14 February 2022

\section{References}

1. Baum A, Kaboli PJ, Schwartz MD. Reduced in-person and increased telehealth outpatient visits during the COVID-19 pandemic. Ann Intern Med. 2021:174(1):129-31.

2. Xu S, Glenn S, Sy L, et al. Impact of the COVID-19 pandemic on health care utilization in a large integrated health care system: retrospective cohort study. J Med Internet Res. 2021;23(4):e26558.

3. Bhatia RS, Chu C, Pang A, Tadrous M, Stamenova V, Cram P. Virtual care use before and during the COVID-19 pandemic: a repeated cross-sectional study. CMAJ Open. 2021;9(1):E107-14.

4. Alexander GC, Tajanlangit M, Heyward J, Mansour O, Qato DM, Stafford RS. Use and content of primary care office-based vs telemedicine care visits during the COVID-19 pandemic in the US. JAMA Netw Open. 2020;3(10):e2021476.

5. Chu C, Cram P, Pang A, Stamenova V, Tadrous M, Bhatia RS. Rural telemedicine use before and during the COVID-19 pandemic: repeated cross-sectional study. J Med Internet Res. 2021;23(4):e26960.

6. Stamenova V, Agarwal P, Kelley L, et al. Uptake and patient and provider communication modality preferences of virtual visits in primary care: a retrospective cohort study in Canada. BMJ Open. 2020;10(7):e037064.

7. Sim I. Mobile devices and health. N Engl J Med. 2019:381(10):956-68.
8. Canadian Medical Association. Virtual care in Canada: discussion paper. Available at: https://www.cma.ca/sites/default/files/pdf/News/Virtual Care_discussionpaper_v2EN.pdf. Accessed 23 June 2021.

9. Almathami HKY, Win KT, Vlahu-Gjorgievska E. Barriers and facilitators that influence telemedicine-based, real-time, online consultation at patients' homes: systematic literature review. J Med Internet Res. 2020;22(2):e16407.

10. Glazier RH, Green ME, Wu FC, Frymire E, Kopp A, Kiran T. Shifts in office and virtual primary care during early COVID-19 pandemic in Ontario, Canada. CMAJ. 2021;193(6):E200-10.

11. Lonergan PE, Washington SL, Branagan L, et al. Rapid utilization of telehealth in a Comprehensive Cancer Center as a response to COVID-19: cross-sectional analysis. J Med Internet Res. 2020;22(7):e19322.

12. Wosik J, Clowse MEB, Overton R, et al. Impact of the COVID-19 pandemic on patterns of outpatient cardiovascular care. Am Heart J. 2021;231:1-5.

13. Billings J, Zeitel L, Lukomnik J, Carey TS, Blank AE, Newman L. Impact of socioeconomic status on hospital use in new York City. Health Aff (Millwood). 1993;12(1):162-73.

14. Zoom Video Communications, Inc. Zoom. 2021. Available at: https:// zoom.us. Accessed 30 June 2021

15. Women's College Hospital. My Health Record Portal. 2021. Available at: https://www.womenscollegehospital.ca/patients-and-caregivers/yourhealth-information/myhealthrecord. Accessed 30 June 2021.

16. Helfenstein $U$. The use of transfer function models, intervention analysis and related time series methods in epidemiology. Int J Epidemiol. 1991:20(3):808-15. https://doi.org/10.1093/ije/20.3.808.

17. Weiner JP, Bandeian S, Hatef E, Lans D, Liu A, Lemke KW. In-person and telehealth ambulatory contacts and costs in a large US insured cohort before and during the COVID-19 pandemic. JAMA Netw Open. 2021:4(3):e212618.

18. Commonwealth Fund. The Impact of the COVID-19 Pandemic on Outpatient Visits: A Rebound Emerges. Available at: https://www.commo nwealthfund.org/publications/2020/apr/impact-covid-19-outpatientvisits. Accessed 23 June 2021

19. Koonin LM, Hoots B, Tsang CA, et al. Trends in the use of telehealth during the emergence of the COVID-19 pandemic — United States, January-March 2020. MMWR Morb Mortal Wkly Rep. 2020;69(430):1595-9.

20. Patel SY, Mehrotra A, Huskamp HA, Uscher-Pines L, Ganguli I, Barnett ML. Trends in outpatient care delivery and telemedicine during the COVID-19 pandemic in the US. JAMA Intern Med. 2021;181(3):388-91.

21. Ferguson JM, Jacobs J, Yefimova M, Greene L, Heyworth L, Zulman DM. Virtual care expansion in the veterans health administration during the COVID-19 pandemic: clinical services and patient characteristics associated with utilization. J Am Med Inform Assoc. 2021;28(3):453-62.

22. Granata T, Bisulli F, Arzimanoglou A, Rocamora R. Did the COVID-19 pandemic silence the needs of people with epilepsy? Epileptic Disord. 2020;22(4):439-42.

23. Michalowsky B, Hoffmann W, Bohlken J, Kostev K. Effect of the COVID19 lockdown on disease recognition and utilisation of healthcare services in the older population in Germany: a cross-sectional study. Age Ageing. 2021;50(2):317-25.

24. Fersia O, Bryant S, Nicholson R, et al. The impact of the COVID-19 pandemic on cardiology services. Open Heart. 2020;7(2):e001359.

25. Doolub G, Wong C, Hewitson L, et al. Impact of COVID-19 on inpatient referral of acute heart failure: a single-Centre experience from the south-west of the UK. ESC Heart Fail. 2021;8(2):1691-5.

26. Czeisler ME, Marynak K, Clarke KEN, et al. Delay or avoidance of medical care because of COVID-19-related concerns - United States, June 2020 MMWR Morb Mortal Wkly Rep. 2020;69(36):1250-7.

27. Findling MG, Blendon RJ, Benson JM. Delayed care with harmful health consequences - reported experiences from national surveys during coronavirus disease 2019. JAMA Netw Open. 2020;1(12):e201463.

28. Appireddy R, Khan S, Leaver C, et al. Home virtual visits for outpatient follow-up stroke care: a cross-sectional study. J Med Internet Res. 2019:21(10):e13734.

29. Gomez T, Anaya TB, Shih KJ, Tarn DM. A qualitative study of primary care physicians' experiences with telemedicine during COVID-19. J Am Board Fam Med. 2021;34(Suppl):S61-70. 
30. Tenforde AS, laccarino MA, Borgstrom H, et al. Telemedicine during COVID-19 for outpatient sports and musculoskeletal medicine physicians. PM R. 2020;12(9):926-32.

31. Eberly LA, Kallan MJ, Julien HM, et al. Patient characteristics associated with telemedicine access for primary and specialty ambulatory care during the COVID-19 pandemic. JAMA Netw Open. 2020;3(12):e2031640.

32. Kochar B, Ufere NN, Nipp R, Gustafson JL, Carolan P, Ritchie CS. Videobased telehealth vistis decrease with increasing age. Am J Gastroenterol. 2021;116(2):431-2.

33. Dewar S, Lee PG, Suh TT, Min L. Uptake of virtual visits in a geriatric primary care clinic during the COVID-19 pandemic. J Am Geriatr Soc. 2020;68(7):1392-4.

34. Levine DM, Lipsitz SR, Linder JA. Trends in seniors' use of digital health technology in the United States, 2011-2014. JAMA. 2016;316(5):538-40.

35. Anderson $M$, Perrin A. Tech adoption climbs among older adults. Published May 17, 2017. Pew Research Center. Available at: https://www. pewresearch.org/internet/2017/05/17/technology-use-among-seniors/. Accessed 23 June 2021.

36. Darrat I, Tam S, Boulis M, Williams AM. Socioeconomic disparities in patient use of telehealth during the coronavirus disease 2019 surge. JAMA Otolaryngol Head Neck Surg. 2021;147(3):287-95.

37. Weber E, Miller SJ, Astha V, Janevic T, Benn E. Characteristics of telehealth users in NYC for COVID-related care during the coronavirus pandemic. J Am Med Inform Assoc. 2020;27(12):1949-54.

\section{Publisher's Note}

Springer Nature remains neutral with regard to jurisdictional claims in published maps and institutional affiliations.

- fast, convenient online submission

- thorough peer review by experienced researchers in your field

- rapid publication on acceptance

- support for research data, including large and complex data types

- gold Open Access which fosters wider collaboration and increased citations

- maximum visibility for your research: over $100 \mathrm{M}$ website views per year

At BMC, research is always in progress.

Learn more biomedcentral.com/submissions 